\title{
An Infrared Multiobject Fiber-Fed Spectrograph for the Calar Alto Observatory
}

\author{
T. M. Herbst, E. Pitz, C. M. Reuther \\ Max-Planck-Institut für Astronomie, Königstuhl 17, 69117 Heidelberg, \\ Germany
}

\begin{abstract}
We report the first use of a near infrared multiobject spectrograph that can acquire 1-2.5 $\mu \mathrm{m}$ spectra of up to 35 objects in a 1000 arcsecond field.
\end{abstract}

\section{Introduction}

Recent technological advances have revolutionized observations in the near infrared, and instruments based on optical fibers are poised to make a significant impact in several fields of astronomy. This paper presents technical details and initial observational results for a new infrared fiber-fed multiobject spectrograph based at the $3.5 \mathrm{~m}$ telescope on Calar Alto, Spain.

\section{The Instrument}

The spectrograph combines the Spaltspinne ("slit spider") fiber positioning device with the MAGIC infrared camera (Pitz 1993, Herbst et al. 1993). Figure 1 is a schematic drawing of the optical path. The fibers are only $60 \mathrm{~cm}$ long, resulting in minimal focal ratio degradation and good transmission over the $\mathrm{J}(1.13-1.37 \mu \mathrm{m}), \mathrm{H}(1.5-1.8 \mu \mathrm{m})$, and $\mathrm{K}(2.0-2.4 \mu \mathrm{m})$ photometric bands. The grism is a resin replica design, with a Milton-Roy 150 line $\mathrm{mm}^{-1}$ grating bonded to the hypotenuse of a prism fabricated of Ultran 30 (Schott). The grism gives spectral resolution $\frac{\lambda}{\Delta \lambda} \sim 200$ and permits simultaneous $H$ and $K$ band coverage in first order and $J$ band coverage in second order. Measurements in the lab and on the telescope indicate that the transmission of the grism exceeds $\mathbf{7 5 \%}$.

\section{First Observations}

We commissioned the spectrograph in January 1994 at the $3.5 \mathrm{~m}$ telescope on Calar Alto, Spain. The $400 \mu \mathrm{m}$ core of the fibers corresponds to a 2.4 aresec aperture at this telescope. Figure 2 shows a typical 10 second exposure of the Orion stellar cluster. The wavelength ranges from $1.5 \mu \mathrm{m}$ on the left to $2.4 \mu \mathrm{m}$ on the right. Approximately 25 distinct stellar spectra appear in the image, with the balance of the fibers being assigned to empty sky. An equal duration off-source exposure has been subtracted to remove airglow lines and thermal emission. The remnant excess noise appears at the longest wavelengths. Figure 3 contains a 


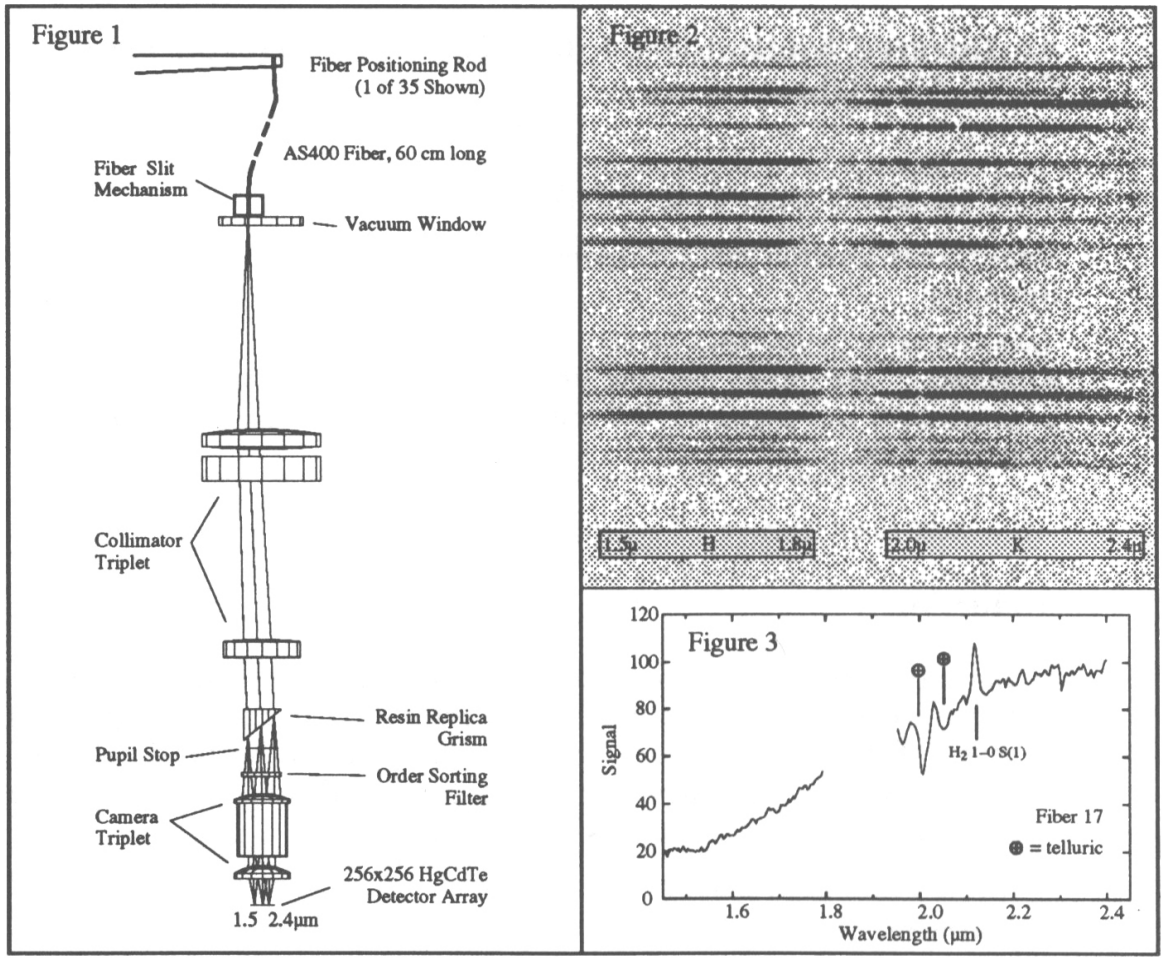

typical spectrum extracted from the average of $\sim 100$ such exposures. These spectra will form part of the dataset used in an ongoing program to analyze the stars in embedded infrared clusters.

\section{Conclusions}

Poor weather conditions prevented an assessment of the instrument's throughput on the telescope in 1994 January. Nevertheless, we identified several modifications to the Spaltspinne / MAGIC fiber-fed infrared spectrograph which will improve its performance in the coming months, and we plan to have the instrument in regular operation for observing programs at Calar Alto by mid-summer 1994.

\section{References}

Herbst, T. M., Beckwith, S. V. W., Birk, Ch., Hippler, S., McCaughrean, M. J., Mannucci, F., and Wolf, J. 1993a, in Infrared Detectors and Instrumentation SPIE Conference 1946.

Pitz, E. 1993, in Fiber Optics in Astronomy II, ASP Conference series No. 37, page 20 . 